\title{
Differentiability from the representation formula and the Sobolev-Poincaré inequality
}

\author{
by \\ Valentino Magnani (Pisa)
}

\begin{abstract}
In the geometries of stratified groups, we provide differentiability theorems for both functions of bounded variation and Sobolev functions. Proofs are based on a systematic application of the Sobolev-Poincaré inequality and the so-called representation formula.
\end{abstract}

Introduction. The aim of the present paper is to introduce a simple and unified approach to higher order differentiability of both Sobolev and BV functions on stratified groups. These groups represent a large family of different geometries, which also include Euclidean spaces [17]. For instance, the Heisenberg group is a well known example of a noncommutative stratified group [42].

The classical notions of Sobolev functions and of functions with bounded variation extend to the general context of stratified groups as follows. We consider left invariant vector fields $X_{1}, \ldots, X_{m}$ and we say that a locally summable function $u$ on an open subset $\Omega$ of a stratified group $\mathbb{G}$ has $H$-bounded variation if its distributional derivative $X_{j} u$ is a finite Radon measure for every $j=1, \ldots, m$. If all distributional derivatives $X_{j_{1}} \cdots X_{j_{p}} u$ are measures for every $p \leq k$ and $j_{s} \in\{1, \ldots, m\}$ for every $s=1, \ldots, p$, then we say that $u$ has $H$-bounded $k$-variation, and write $u \in \operatorname{BV}_{\mathrm{H}}^{k}(\Omega)$. The H-Sobolev space $W_{\mathrm{H}}^{k, p}(\Omega)$ is defined in analogous way; the central role played by the vector fields $X_{j}$ is then clear. In fact, they span the first layer of the stratified algebra and yield the geometry of the group, defining the so-called Carnot-Carathéodory distance (see Section 1 below).

In order to state our results, we introduce the notion of " $L^{p}$ differentiability of order $k$ ". In the Euclidean context this definition goes back to Calderón and Zygmund [6], [7]. A function $u \in L_{\text {loc }}^{p}(\Omega)$ is said to be $L^{p}$ differentiable of order $k$ at $x \in \Omega$ if there exists a polynomial $P_{[x]}$ of homogeneous degree less than or equal to $k$ such that

2000 Mathematics Subject Classification: Primary 26B05; Secondary 22E30. 


$$
\left(f_{B_{x, r}}\left|u(y)-P_{[x]}(y)\right|^{p} d y\right)^{1 / p}=o\left(r^{k}\right) \quad \text { as } r \rightarrow 0^{+} .
$$

The anisotropy of stratified groups requires the notion of homogeneous degree of a polynomial, which amounts to take into account the "weight" of every coordinate (see Subsection 1.2). The polynomial $P_{[x]}$ is uniquely defined and it is called the " $L^{p}$ differential of $u$ of order $k$ ". Note that every function $u$ which is $L^{p}$ differentiable of order $k$ at $x \in \Omega$ satisfies the equivalent condition

$$
\lim _{r \rightarrow 0^{+}} \underset{B_{x, r}}{f}\left(\frac{\left|u(y)-P_{[x]}(y)\right|}{\varrho(x, y)^{k}}\right)^{p} d y=0,
$$

as we show in Lemma 1.16. It is well known that $L^{p}$ differentiability turns out to be the natural concept to study fine properties of functions with bounded variation and Sobolev functions in Euclidean spaces. In this case $L^{p}$ differentiability and approximation theorems for Sobolev functions can be found for instance in [2], [4]-[7], [15]; see also [41], [46] and the references therein.

Previous results on higher order differentiability in stratified groups can be found in [1]. The importance of higher order differentiability stems for instance from the recent interest in studying second order pointwise differentiability of H-convex functions [13], [24], [26], [34], [36]. The present paper can be thought of as a development of [1]; we extend the previous results adopting a simpler approach which also applies to H-Sobolev functions. Here we do not use maximal functions and Rademacher's theorem. On the contrary, as a byproduct of our results and of Lemma 3.1.5 in [16], we also provide another proof of Rademacher's theorem on stratified groups.

Our first order differentiability theorem is stated as follows. Let $u: \Omega \rightarrow$ $\mathbb{R}$ be a function of $\mathrm{H}$-bounded variation and let $\beta=Q /(Q-1)$, where $Q$ is the Hausdorff dimension of the group. Then $u$ is a.e. $L^{\beta}$ differentiable and its differential corresponds to the density of $D_{\mathrm{H}} u^{\mathrm{a}}$, which is the absolutely continuous part of the the measure $D_{\mathrm{H}} u$ (see Section 1 for precise definitions). The Euclidean version of this result has been first proved by Calderón and Zygmund [6] (see also Federer's proof in Theorem 4.5.9(26) of [16]). First order differentiability results for Sobolev functions with $1<p<\infty$ are already known in the general setting of doubling metric spaces admitting the Poincaré inequality [3]. Our method for first order differentiability also applies to Sobolev functions for any $p \geq 1$. The main features of our approach consist in the use of the Sobolev-Poincare inequality (9) and the so-called "representation formula", stated as follows. There exists a dimensional constant $C$ such that for every function $u$ in $C^{\infty}(\Omega)$ and every open ball $B_{x, 2 r}$ 
compactly contained in $\Omega$, the pointwise estimate

$$
\left|u(x)-u_{B_{x, r}}\right| \leq C \int_{B_{x, r}} \frac{\left|\nabla_{\mathrm{H}} u(y)\right|}{\varrho(x, y)^{Q-1}} d y
$$

holds, where $\varrho$ is the Carnot-Carathéodory distance (see Section 1 for more details). This formula on stratified groups has been proved by Lu [31] (see also [10], [18], [19] and [35]).

The core of the present paper pertains to higher order differentiability, which poses novel difficulties, due to the noncommutativity of the vector fields $X_{1}, \ldots, X_{m}$. In fact, the characterization of the differential $P_{[x]}$ in (1) using distributional derivatives of $u$ requires a basis of left invariant differential operators on $\mathbb{G}$. By the Poincaré-Birkhoff-Witt theorem one can explicitly construct such a basis by iterated compositions of $X_{1}, \ldots, X_{m}$, seen as first order left invariant differential operators. As a consequence, Proposition 3.1 provides a characterization of $P_{[x]}$. This proposition plays a crucial role in the argument by induction which connects first order differentiability to higher order differentiability. We show that every function $u \in \operatorname{BV}_{\mathrm{H}}^{k}(\Omega)$ is $L^{1}$ differentiable of order $k$ and that in the case $k<Q$ it is $L^{\gamma}$ differentiable of the same order, where $\gamma=Q /(Q-k)$. In Euclidean spaces this result fits in Theorem 1(i) of [15]. Note that the Euclidean proof of [15] uses minimizing polynomials, which suitably replace the Taylor expansion of the function, hence following a completely different approach. In analogous way, we prove that every function $u$ in $W_{\mathrm{H}}^{k, p}(\Omega)$ is a.e. $L^{p}$ differentiable of order $k$ and that in the case $k p<Q$ it is a.e. $L^{\eta}$ differentiable, where $\eta=Q p /(Q-k p)$.

Finally, we give a brief overview of the present paper. Section 1 is devoted to preliminary notions and basic tools used in the subsequent sections. We prove Lemma 1.16 and we give a simple argument leading to the key estimates (20) and (21). In Section 2 we prove the sub-Riemannian version of the well known Calderón-Zygmund differentiability of functions with bounded variation. As a byproduct of our first order differentiability results, we give another proof of the Rademacher theorem on stratified groups. In Section 3 we prove higher order differentiability of functions in $\operatorname{BV}_{\mathrm{H}}^{k}(\Omega)$ and in $W_{\mathrm{H}}^{k, p}(\Omega)$.

Acknowledgements. I am glad to thank Daniele Morbidelli for a fruitful discussion during the "Workshop on Analysis and Geometry in CarnotCarathéodory spaces" held at Arkansas University on March 7-8th, 2003. I also thank the organizer Luca Capogna for his kind invitation. It is a pleasure to thank Luigi Ambrosio for his valuable suggestions. I wish to thank Giovanni Alberti and Jan Malý for calling my attention to Dorronsoro's paper. 
1. Preliminaries. A stratified group is a simply connected nilpotent Lie group $\mathbb{G}$ endowed with a graded Lie algebra $\mathcal{G}$, which is decomposed into a direct sum of subspaces $V_{j}$ subject to the condition $V_{j+1}=\left[V_{j}, V_{1}\right]$ for every $j \in \mathbb{N} \backslash\{0\}$, and $V_{j}=\{0\}$ whenever $j$ is greater than a positive integer. We denote by $\iota$ the maximum integer such that $V_{\iota} \neq\{0\}$ and we call it the nilpotence degree or the step of the group. Recall that for arbitrary subspaces $V, W \subset \mathcal{G}$ we define $[V, W]=\operatorname{span}\{[X, Y] \mid X \in V, Y \in W\}$.

The assumption that $\mathbb{G}$ is simply connected and nilpotent ensures that the exponential map exp $: \mathcal{G} \rightarrow \mathbb{G}$ is a diffeomorphism. The grading of $\mathcal{G}$ allows us to define dilations on the group, i.e. maps $\delta_{t}: \mathcal{G} \rightarrow \mathcal{G}$ with $\delta_{t}\left(\sum_{j=1}^{\iota} v_{j}\right)=\sum_{j=1}^{\iota} t v_{j}$, where $t>0$ and $\sum_{j=1}^{\iota} v_{j} \in \mathcal{G}$ is the unique representation of a vector of $\mathcal{G}$ with $v_{j} \in V_{j}$ for every $j=1, \ldots, \iota$. This notion of dilation is motivated by the fact that the composition exp $\circ \delta_{t} \circ \ln : \mathbb{G} \rightarrow \mathbb{G}$ is a group homomorphism, where $\ln =\exp ^{-1}$. We will use the same symbol to denote dilations which are read on the group. The underlying metric of the group is a left invariant Riemannian metric $g$ such that the subspaces $V_{j}$ are orthogonal to each other. We will always refer to these metrics, called graded metrics. The Riemannian volume on $\mathbb{G}$ given by a graded metric will be denoted by $v_{g}$. It is clear that $v_{g}$ is left invariant, hence it is a Haar measure of the group. For ease of notation, we will also write $v_{g}(A)=|A|$, where $A \subset \mathbb{G}$ is a measurable set, and we will use the symbol $d x$ when integration is considered with respect to the Riemannian volume measure $v_{g}$. The averaged integral of a summable map $u: A \rightarrow \mathbb{R}$ is defined as $u_{A}=f_{A} u=|A|^{-1} \int_{A} u$.

The Carnot-Carathéodory distance between two points $x$ and $x^{\prime}$ is obtained by taking the infimum of the lengths of absolutely continuous curves a.e. tangent to the horizontal subbundle and connecting $x$ with $x^{\prime}$. The length of connecting curves is computed by a graded metric $g$, hence the Carnot-Carathéodory distance $\varrho$ is left invariant and it has the important scaling property $\varrho\left(\delta_{t} x, \delta_{t} x^{\prime}\right)=t \varrho\left(x, x^{\prime}\right)$, where $x, x^{\prime} \in \mathbb{G}$ (see for instance [25]). We denote by $B_{x, r}$ the open ball of center $x$ and radius $r>0$ with respect to the Carnot-Carathéodory distance. Balls of radius $r$ centered at the unit element $e$ of the group will be denoted simply by $B_{r}$. We will frequently use the following scaling property:

$$
\left|B_{x, r}\right|=\left|B_{1}\right| r^{Q}
$$

for every $x \in \mathbb{G}$ and any $r>0$. The integer $Q$ is the Hausdorff dimension of $\mathbb{G}$ with respect to the Carnot-Carathéodory distance and it is strictly greater than the topological dimension $q$ of the group whenever $\mathbb{G}$ is not Abelian. More information on stratified groups can be found for instance in [12], [17] and [42]. 
We introduce the horizontal space $\mathrm{H}_{p} \mathbb{G}=\left\{X(p) \mid X \in V_{1}\right\}$ at $p \in \mathbb{G}$ and consider the disjoint union of all these subspaces with the relevant vector bundle topology. This collection forms the so-called horizontal subbundle, denoted by $\mathrm{H} \mathbb{G}$. Smooth sections of the horizontal subbundle are called horizontal vector fields. We assume throughout the paper that $\Omega$ is an open subset of a stratified group $\mathbb{G}$. The space of smooth horizontal vector fields of $\Omega$ is denoted by $\Gamma(\mathrm{H} \Omega)$ and the one of compactly supported horizontal vector fields by $\Gamma_{\mathrm{c}}(\mathrm{H} \Omega)$. Note that a horizontal vector field $\varphi$ can be written as $\sum_{j=1}^{m} \varphi_{j} X_{j}$, where $\left(X_{1}, \ldots, X_{m}\right)$ is a basis of the first layer $V_{1} \subset \mathcal{G}$. The $H$-divergence of $\varphi$ is defined as $\sum_{j=1}^{m} X_{j} \varphi_{j}$ and it is denoted by $\operatorname{div}_{\mathrm{H}} \varphi$. Note that the definition of $\mathrm{H}$-divergence does not depend on the choice of the basis of $V_{1}$. These notions allow us to introduce functions of bounded variations naturally associated to the sub-Riemannian structure of the group (see [9], [20] for more information).

DEFinition 1.1 (H-BV functions). We say that a function $u \in L^{1}(\Omega)$ is a function of $H$-bounded variation (for short, an $H$ - $B V$ function) if

$$
\left|D_{\mathrm{H}} u\right|(\Omega):=\sup \left\{\int_{\Omega} u(x) \operatorname{div}_{\mathrm{H}} \varphi(x) d x\left|\varphi \in \Gamma_{\mathrm{c}}(\mathrm{H} \Omega),\right| \varphi \mid \leq 1\right\}<\infty .
$$

We denote by $\mathrm{BV}_{\mathrm{H}}(\Omega)$ and $\mathrm{BV}_{\mathrm{H}, \text { loc }}(\Omega)$ the space of functions of $\mathrm{H}$-bounded variation and of locally $\mathrm{H}$-bounded variation, respectively.

By the Riesz representation theorem we get the existence of a nonnegative Radon measure $\left|D_{\mathrm{H}} u\right|$ and of a Borel section $\nu$ of $\mathrm{H} \Omega$ such that $|\nu(x)|=1$ for $\left|D_{\mathrm{H}} u\right|$-a.e. $x \in \Omega$. Moreover for every $\phi \in \Gamma_{\mathrm{c}}(\mathrm{H} \Omega)$ the following integration by parts formula holds:

$$
\int_{\Omega} u(x) \operatorname{div}_{\mathrm{H}} \phi(x) d x=-\int_{\Omega}\langle\phi, \nu\rangle d\left|D_{\mathrm{H}} u\right| .
$$

The vector-valued measure $\nu\left|D_{\mathrm{H}} u\right|$ is denoted by $D_{\mathrm{H}} u$. The symbols $\left|D_{\mathrm{H}} u\right|^{\text {a }}$ and $\left|D_{\mathrm{H}} u\right|^{\mathrm{s}}$ stand for the absolutely continuous part and the singular part of $\left|D_{\mathrm{H}} u\right|$, respectively. We also define the vector measures $D_{\mathrm{H}}^{\mathrm{a}} u=\nu\left|D_{\mathrm{H}} u\right|^{\mathrm{a}}$ and $D_{\mathrm{H}}^{\mathrm{s}} u=\nu\left|D_{\mathrm{H}} u\right|^{\mathrm{s}}$.

The density of $D_{\mathrm{H}}^{\mathrm{a}} u$ with respect to the Haar measure of the group is denoted by $\nabla_{\mathrm{H}} u$. In our arguments we will consider $\nabla_{\mathrm{H}} u$ as a measurable vector function with values in $\mathbb{R}^{m}$. This is possible whenever we refer to an orthonormal basis $\left(X_{1}, \ldots, X_{m}\right)$ of $V_{1}$. Note that if $u$ is of class $C^{1}$, then $\nabla_{\mathrm{H}} u=\left(X_{1} u, \ldots, X_{m} u\right)$.

DEFinition 1.2 (H-BV ${ }^{k}$ functions). By induction on $k \geq 2$ we say that a measurable map $u: \Omega \rightarrow \mathbb{R}$ has $H$-bounded $k$-variation (for short, an $\mathrm{H}-\mathrm{BV}^{k}$ function) if for any $i=1, \ldots, m$ the distributional derivatives $X_{i} u$ 
are representable by functions with $\mathrm{H}$-bounded $(k-1)$-variation. We denote by $\operatorname{BV}_{\mathrm{H}}^{k}(\Omega)$ the space of all $\mathrm{H}-\mathrm{BV}^{k}$ functions.

Definition 1.3 (H-Sobolev functions). A function $u \in L^{p}(\Omega)$ belongs to $W_{\mathrm{H}}^{k, p}(\Omega)$ if for all $j_{s} \in\{1, \ldots, m\}$ and $s=1, \ldots, k$ there exists a function $v_{j_{1}, \ldots, j_{k}} \in L^{p}(\Omega)$ such that

$$
\int_{\Omega} u(y)\left(X_{j_{1}} \cdots X_{j_{k}} \phi\right)(y) d y=(-1)^{k} \int_{\Omega} v_{j_{1}, \ldots, j_{k}}(y) \phi(y) d y
$$

for every $\phi \in C_{\mathrm{c}}^{\infty}(\Omega)$, where $\left(X_{1}, \ldots, X_{m}\right)$ is an orthonormal basis of $V_{1}$. We denote by $W_{\mathrm{H}, \text { loc }}^{k, p}(\Omega)$ the space of measurable functions defined on $\Omega$ which belong to $W_{\mathrm{H}}^{k, p}\left(\Omega^{\prime}\right)$ for every open set $\Omega^{\prime}$ compactly contained in $\Omega$.

REMARK 1.4. We observe that Definitions 1.2 and 1.3 do not depend on the choice of the orthonormal basis $\left(X_{1}, \ldots, X_{m}\right)$.

1.1. Poincaré and Sobolev-Poincaré inequalities. Important tools for our study are the Poincare inequality and the Sobolev-Poincaré inequality on stratified groups. These inequalities hold for smooth functions on more general Carnot-Carathéodory spaces endowed with a system of Hörmander vector fields. As pointed out in [32], Jerison's result [28] includes the Poincaré inequality with respect to the $L^{p}$ norm for every $p \in[1, \infty[$. Another approach to the Poincaré inequality for vector fields has recently been developed by Lanconelli and Morbidelli [29]. In the case of Lie groups a simple proof of the Poincaré inequality has been given by Varopoulos [43] (see also [27] and [40]).

THEOREM 1.5 (Poincaré inequality). Let $\Omega$ be an open subset of $\mathbb{G}$ and let $1 \leq p<\infty$. Then there exists a constant $C>0$ such that for every $u \in C^{\infty}(\Omega)$ and any ball $B_{x, 2 r}$ compactly contained in $\Omega$ we have

$$
\int_{B_{x, r}}\left|u(y)-u_{B_{x, r}}\right|^{p} d y \leq C r^{p} \int_{B_{x, r}}\left|\nabla_{\mathrm{H}} u(y)\right|^{p} d y .
$$

The following Sobolev-Poincaré inequality has been proved in [18], [31] and [37].

THEOREM 1.6 (Sobolev-Poincaré inequality). Let $\Omega$ be an open subset of $\mathbb{G}$ and let $1 \leq p<Q$. Then there exists a constant $C>0$ such that for every $u \in C^{\infty}(\Omega)$, setting $\gamma=Q p /(Q-p)$, we have

$$
\left(f_{B_{x, r}}\left|u(y)-u_{B_{x, r}}\right|^{\gamma} d y\right)^{1 / \gamma} \leq C r\left(f_{B_{x, r}}\left|\nabla_{\mathrm{H}} u(y)\right|^{p} d y\right)^{1 / p} .
$$

Smooth approximation of functions of bounded variation is also available in general Carnot-Carathéodory spaces [20], [22]; then the previous SobolevPoincare inequality in the case $p=1$ can be extended to H-BV functions. 
THEOREM 1.7. Let $\Omega$ be an open subset of $\mathbb{G}$ and let $\beta=Q /(Q-1)$. Then there exists a constant $C>0$ such that for every $u \in \operatorname{BV}_{\mathrm{H}, \mathrm{loc}}(\Omega)$ and any ball $B_{x, 2 r}$ compactly contained in $\Omega$ we have

$$
\left(f_{B_{x, r}}\left|u(z)-u_{B_{x, r}}\right|^{\beta} d z\right)^{1 / \beta} \leq C r \frac{\left|D_{\mathrm{H}} u\right|\left(B_{x, r}\right)}{\left|B_{x, r}\right|} .
$$

Note that as a consequence of (9) we have the following Poincaré inequality for H-BV functions:

$$
\int_{B_{x, r}}\left|u(z)-u_{B_{x, r}}\right| d z \leq C r\left|D_{\mathrm{H}} u\right|\left(B_{x, r}\right) .
$$

1.2. Approximate regularity and $L^{p}$ differentiability of order $k$. In this subsection we introduce several notions of weak regularity for measurable functions on stratified groups. We begin by recalling the notion of approximate continuity.

DEFINITION 1.8 (Approximate continuity). We say that $u \in L_{\text {loc }}^{1}\left(\Omega, \mathbb{R}^{m}\right)$ has an approximate limit $\lambda \in \mathbb{R}^{m}$ at $x \in \Omega$ if

$$
\lim _{r \rightarrow 0^{+}} f_{B_{x, r}}|u(y)-\lambda| d y=0 .
$$

In the case $\lambda=u(x)$ we say that $x$ is an approximate continuity point and we denote by $A_{u}$ the subset of all approximate continuity points of $u$.

REMARK 1.9. Notice that the approximate limit is uniquely defined and it does not depend on the representative element of $u$. In the next proposition we recall $p$-approximate continuity of functions in $L^{p}(\Omega)$ using the doubling property of $\mathbb{G}$. As a consequence of this result for $p=1$ we have $\left|\Omega \backslash A_{u}\right|=0$.

Proposition 1.10. Let $u \in L_{\mathrm{loc}}^{p}\left(\Omega, \mathbb{R}^{m}\right)$ and let $1 \leq p<\infty$. Then for a.e. $x \in \Omega$ we have

$$
\underset{B_{x, r}}{f}|u(y)-u(x)|^{p} d y=0 .
$$

Proof. Stratified groups endowed with their left invariant volume measure are in particular doubling spaces. Therefore Theorem 2.9.8 of [16] is available and it implies that for a.e. $x \in \Omega$ and for every $q \in \mathbb{Q}^{m}$ the following limit exists:

Then we get

$$
\lim _{r \rightarrow 0^{+}} \underset{B_{x, r}}{f}|u(y)-q|^{p} d y=|u(x)-q|^{p}
$$

$$
\limsup _{r \rightarrow 0^{+}}\left(f_{B_{x, r}}|u(y)-u(x)|^{p}\right)^{1 / p} \leq 2|u(x)-q| \rightarrow 0 \quad \text { as } q \rightarrow u(x),
$$

and this completes the proof. 
Definition 1.11. We define $n_{j}=\operatorname{dim} V_{j}$ for any $j=1, \ldots, \iota, m_{0}=0$ and $m_{i}=\sum_{j=1}^{i} n_{j}$ for any $i=1, \ldots, \iota$. We say that a basis $\left(W_{1}, \ldots, W_{q}\right)$ of $\mathcal{G}$ is an adapted basis if

$$
\left(W_{m_{j-1}+1}, \ldots, W_{m_{j}}\right)
$$

is a basis of $V_{j}$ for any $j=1, \ldots, \iota$.

In order to tackle the higher order approximate differentiability of $\mathrm{H}-\mathrm{BV}^{k}$ functions we will recall some well known facts about polynomials on stratified groups. We will refer to Chapter 1.C of [17]. A function $P: \mathbb{G} \rightarrow \mathbb{R}$ is a polynomial on $\mathbb{G}$ if the composition $P \circ \exp$ is a polynomial on $\mathcal{G}$. Let us fix an adapted basis $\left(W_{1}, \ldots, W_{q}\right)$ and its dual basis of 1 -forms $\left(\eta_{1}, \ldots, \eta_{q}\right)$. Then every polynomial $P: \mathbb{G} \rightarrow \mathbb{R}$ can be represented as

$$
P=\sum_{\alpha} c_{\alpha} x^{\alpha},
$$

where $\left(x_{1}, \ldots, x_{q}\right)$ is a fixed coordinate system on $\mathbb{G}$ given by $x_{i}=\eta_{i} \circ \exp ^{-1}$ for $i=1, \ldots, q$, we set $x^{\alpha}=x_{1}^{\alpha_{1}} \cdots x_{q}^{\alpha_{q}}$ for every $\alpha \in \mathbb{N}^{q}$, and only a finite number of coefficients $c_{\alpha} \in \mathbb{R}$ do not vanish.

The degree $d_{i}$ of $W_{i}$ is well defined by the relation $W_{i} \in V_{d_{i}}$. Then we define the homogeneous degree of a polynomial $P: \mathbb{G} \rightarrow \mathbb{R}$ with expression (13) as

$$
\text { h-deg }(P)=\max \left\{d(\alpha) \mid c_{\alpha} \neq 0\right\}
$$

where $d(\alpha)=\sum_{k=1}^{q} d_{k} \alpha_{k}$. The space of polynomials of homogeneous degree less than or equal to $k$ will be denoted by $\mathcal{P}_{\mathrm{H}, k}(\mathbb{G})$.

Next, we extend the definition of $L^{p}$ differentiability of order $k$ to stratified groups. This notion is due to Calderón and Zygmund (see [6] and [7]).

DEFinition 1.12 ( $L^{p}$ differentiability of order $k$ ). A function $u \in L_{\mathrm{loc}}^{1}(\Omega)$ is said to be $L^{p}$ differentiable of order $k$ at $x \in \Omega$ if there exists a polynomial $T_{[x]} \in \mathcal{P}_{\mathrm{H}, k}(\mathbb{G})$ such that

$$
\left(f_{B_{x, r}}\left|u(y)-T_{[x]}(y)\right|^{p} d y\right)^{1 / p}=o\left(r^{k}\right) \quad \text { as } r \rightarrow 0^{+} .
$$

REMARK 1.13. The $L^{p}$ differential of order $k$ is always uniquely defined. In fact, a function $f$ which is $L^{p}$ differentiable of order $k$ at $x$ is also $L^{1}$ differentiable, since

$$
f_{B_{x, r}}\left|u(y)-T_{[x]}(y)\right| d y \leq\left(f_{B_{x, r}}\left|u(y)-T_{[x]}(y)\right|^{p} d y\right)^{1 / p},
$$

where $1 \leq p<\infty$. Then Lemma 1.14 implies uniqueness. Similarly, one can check that more generally $L^{p}$ differentiability of order $k$ implies $L^{r}$ differentiability of order $k$ at the same point for all $1 \leq r \leq p$. 
Lemma 1.14. Let $P \in \mathcal{P}_{\mathrm{H}, k}(\mathbb{G})$ be such that

$$
\lim _{r \rightarrow 0^{+}} f_{B_{x, r}} \frac{|P(y)|}{r^{k}} d y=0 .
$$

Then $P$ is the null polynomial.

Proof. By a left translation at the unit element of the group we can assume that $x=e$. We fix a coordinate system $\left(x_{1}, \ldots, x_{q}\right)$ on $\mathbb{G}$, hence we can write $P(y)=\sum_{d(\alpha) \leq k} c_{\alpha} y^{\alpha}$. Suppose by contradiction that $P$ is not vanishing. Then the integer $p=\min \left\{d(\alpha) \mid c_{\alpha} \neq 0\right\}$ is well defined and we set $P_{1}(y)=\sum_{d(\alpha)=p} c_{\alpha} y^{\alpha}$. By the homogeneity of $P_{1}$ it follows that

$$
P\left(\delta_{r} y\right)=r^{p}\left(P_{1}(y)+\sum_{d(\alpha)>p} c_{\alpha} r^{d(\alpha)-p} y^{\alpha}\right) .
$$

Defining $Q_{r}(y)=\sum_{d(\alpha)>p} c_{\alpha} r^{d(\alpha)-p} y^{\alpha}$, we note that

$$
\sup _{y \in B_{1}}\left|Q_{r}(y)\right| \rightarrow 0 \quad \text { as } r \rightarrow 0^{+} \text {. }
$$

By a change of variable we have

$$
f_{B_{r}} \frac{|P(y)|}{r^{k}} d y=f_{B_{1}} \frac{\left|P\left(\delta_{r} y\right)\right|}{r^{k}} d y \rightarrow 0 \quad \text { as } r \rightarrow 0^{+} .
$$

Then for a sufficiently small $r \in] 0,1$ [ we obtain

$$
f_{B_{1}} \frac{\left|P\left(\delta_{r} y\right)\right|}{r^{k}} d y \geq r^{p-k}\left(f_{B_{1}}\left|P_{1}(y)\right| d y-f_{B_{1}}\left|Q_{r}(y)\right| d y\right) \geq \frac{1}{2} f_{B_{1}}\left|P_{1}(y)\right| d y .
$$

Since $P_{1}$ is a nonvanishing polynomial, this inequality contradicts (15).

REMARK 1.15. The next lemma proves that condition (14) is equivalent to

$$
\lim _{r \rightarrow 0^{+}} \underset{B_{x, r}}{f}\left(\frac{\left|u(y)-T_{[x]}(y)\right|}{\varrho(x, y)^{k}}\right)^{p} d y=0 .
$$

LEMMA 1.16. Let $g \in L_{\text {loc }}^{1}(\Omega)$ be such that $g \geq 0$ a.e. in $\Omega$ and let $\alpha>0$. Then the condition $f_{B_{x, r}} g=o\left(r^{\alpha}\right)$ as $r \rightarrow 0^{+}$implies that

$$
\lim _{r \rightarrow 0^{+}} f_{B_{x, r}} \frac{g(y)}{\varrho(x, y)^{\alpha}} d y=0 .
$$

Proof. Let $x \in \Omega$ and let $r>0$ be sufficiently small. We define $r_{k}=r 2^{-k}$ for every integer $k \geq 0$. Then we have 


$$
\begin{aligned}
f_{B_{x, r}} \frac{g(y)}{\varrho(x, y)^{\alpha}} d y & \leq \frac{2^{\alpha}}{\left|B_{x, r}\right|} \sum_{k=0}^{\infty} \frac{1}{r_{k}^{\alpha}} \int_{B_{x, r_{k}}} g(y) d y=2^{\alpha} \sum_{k=0}^{\infty} \frac{2^{-k Q}}{r_{k}^{\alpha}} f_{B_{x, r_{k}}} g(y) d y \\
& \leq \frac{2^{\alpha}}{1-2^{-Q}} \sup _{0<s \leq r} s^{-\alpha} f_{B_{x, s}} g(y) d y \rightarrow 0
\end{aligned}
$$

as $r \rightarrow 0^{+}$.

1.3. Representation formula. Another important tool is the "representation formula" stated in the next theorem. This formula will lead us to the pointwise estimates (20) and (21) that play a key role in our method to obtain differentiability results. In the next theorem we state the representation formula for H-BV functions. Its proof can be easily obtained for instance following the method of [19]. Short proofs of the representation formula can also be found in [9] and [35]. In particular, we mention the sharp result of [35], where, in a more general metric setting, the integral in the representation formula is considered on the ball with optimal radius.

THEOREM 1.17 (Representation formula). Let $\Omega \subset \mathbb{G}$ be an open subset. Then there exists a constant $C>0$ such that for every ball $B_{x, 2 r}$ compactly contained in $\Omega$ we have

$$
\left|u(x)-u_{B_{x, r}}\right| \leq C \int_{B_{x, r}} \frac{1}{\varrho(x, z)^{Q-1}} d\left|D_{\mathrm{H}} u\right|(z)
$$

for every $u \in \mathrm{BV}_{\mathrm{H}, \mathrm{loc}}\left(B_{x, r}\right)$ whenever $x \in B_{x, r} \cap A_{u}$.

We can rewrite (18) using Fubini's theorem as follows. For every $\alpha>1$ we have

$$
\begin{aligned}
& \int_{B_{x, r}} \frac{1}{\varrho(x, z)^{\alpha-1}} d\left|D_{\mathrm{H}} u\right|(z)=(\alpha-1) \int_{B_{x, r}}\left(\int_{\varrho(x, z)}^{\infty} \frac{1}{t^{\alpha}} d t\right) d\left|D_{\mathrm{H}} u\right|(z) \\
& =(\alpha-1) \int_{0}^{\infty} \frac{\left|D_{\mathrm{H}} u\right|\left(B_{x, r} \cap B_{x, t}\right)}{t^{\alpha}} d t=(\alpha-1) \int_{0}^{r} \frac{\left|D_{\mathrm{H}} u\right|\left(B_{x, t}\right)}{t^{\alpha}} d t+\frac{\left|D_{\mathrm{H}} u\right|\left(B_{x, r}\right)}{r^{\alpha-1}}
\end{aligned}
$$

that implies by a change of variable

$$
\int_{B_{x, r}} \frac{1}{\varrho(x, z)^{\alpha-1}} d\left|D_{\mathrm{H}} u\right|(z)=\frac{\alpha-1}{r^{\alpha-1}} \int_{0}^{1} \frac{\left|D_{\mathrm{H}} u\right|\left(B_{x, t r}\right)}{t^{\alpha}} d t+\frac{\left|D_{\mathrm{H}} u\right|\left(B_{x, r}\right)}{r^{\alpha-1}} .
$$

The representation formula along with (19) yields the following theorem.

THEOREM 1.18. Let $\Omega \subset \mathbb{G}$ be a bounded open set. Then there exists a constant $C>0$ such that for every ball $B_{x, 2 r}$ compactly contained in $\Omega$ we 
have

$$
\left|u(x)-u_{B_{x, r}}\right| \leq C r\left\{\int_{0}^{1} \frac{\left|D_{\mathrm{H}} u\right|\left(B_{x, t r}\right)}{(t r)^{Q}} d t+\frac{\left|D_{\mathrm{H}} u\right|\left(B_{x, r}\right)}{r^{Q}}\right\}
$$

for every $u \in \mathrm{BV}_{\mathrm{H}, \mathrm{loc}}(\Omega)$ whenever $x \in B_{x, r} \cap A_{u}$.

As an immediate consequence we have the following corollary.

Corollary 1.19. Let $\Omega \subset \mathbb{G}$ be a bounded open set and let $1 \leq p<\infty$. Then there exists a constant $C>0$ such that for every ball $B_{x, 2 r}$ compactly contained in $\Omega$ we have

$$
\begin{aligned}
\left|u(x)-u_{B_{x, r}}\right| \leq & C r\left\{\int_{0}^{1}\left(f_{B_{x, t r}}\left|\nabla_{\mathrm{H}} u(y)\right|^{p} d y\right)^{1 / p} d t\right. \\
& \left.+\left(f_{B_{x, r}}\left|\nabla_{\mathrm{H}} u(y)\right|^{p} d y\right)^{1 / p}\right\} .
\end{aligned}
$$

for every $u \in W_{\mathrm{H}, \mathrm{loc}}^{1, p}(\Omega)$ whenever $x \in B_{x, r} \cap A_{u}$.

2. First order differentiability. We begin this section with some preliminary lemmas that will be used in what follows. Throughout the paper we will use the notation $v^{*}$ for the map $x \mapsto\langle v, \ln x\rangle$, where $x \in \mathbb{G}$ and $v \in V_{1}$. Note that $v^{*}\left(\delta_{r} x\right)=r v^{*}(x)$ and $v^{*}: \mathbb{G} \rightarrow \mathbb{R}$ is a group homomorphism.

LEMMA 2.1. Let $\mu$ be a nonnegative Radon measure on $\Omega$ such that there exists a subset $N \subset \Omega$ of Haar measure zero and $\mu(\Omega \backslash N)=0$. Then $\lim _{r \rightarrow 0^{+}} \mu\left(B_{x, r}\right) r^{-Q}=0$ for a.e. $x \in \Omega$.

Proof. By contradiction, if we had a measurable subset $A \subset \Omega$ with $|A|>0$ such that

$$
\limsup _{r \rightarrow 0^{+}} \frac{\mu\left(B_{x, r}\right)}{r^{Q}}>0
$$

for every $x \in A$, then we would get a measurable subset $A^{\prime} \subset A \backslash N$ with positive measure and $\lambda>0$ such that $\left|D_{\mathrm{H}}^{\mathrm{s}} u\right|\left(A^{\prime}\right) \geq \lambda \mu\left(A^{\prime}\right)>0$. This standard fact can be checked for instance by Theorems 2.10.17 and 2.10.18 of [16]. Then the inequalities

$$
0<\mu\left(A^{\prime}\right) \leq \mu(\Omega \backslash N)
$$

lead us to a contradiction.

LEMma 2.2. Let $u \in \mathrm{BV}_{\mathrm{H}, \mathrm{loc}}(\Omega)$. Then for a.e. $x \in A_{\nabla_{\mathrm{H}} u}$ and every $c \in \mathbb{R}$,

$$
\lim _{r \rightarrow 0^{+}} \frac{\left|D_{\mathrm{H}}\left(u-\nabla_{\mathrm{H}} u(x)^{*}-c\right)\right|\left(B_{x, r}\right)}{r^{Q}}=0 .
$$


Proof. Using the definitions of the absolutely continuous part and singular part of $\left|D_{\mathrm{H}} u\right|$ one can check without difficulty that

$$
\left|D_{\mathrm{H}}\left(u-\nabla_{\mathrm{H}} u(x)^{*}-c\right)\right|=\left|\nabla_{\mathrm{H}} u-\nabla_{\mathrm{H}} u(x)\right| v_{g}+\left|D_{\mathrm{H}} u\right|^{\mathrm{s}} .
$$

Let us first check that for a.e. $x \in \Omega$ we have

$$
\lim _{r \rightarrow 0^{+}} \frac{\left|D_{\mathrm{H}} u\right|^{\mathrm{s}}\left(B_{x, r}\right)}{r^{Q}}=0 .
$$

The Radon-Nikodým theorem gives us a measurable subset $N \subset \Omega$ such that

$$
|N|=0 \quad \text { and } \quad\left|D_{\mathrm{H}} u\right|^{\mathrm{s}}\left(N^{\mathrm{c}}\right)=0 .
$$

Then by Lemma 2.1 the limit (23) holds a.e. in $\Omega$. As a consequence, the assumption that $x$ is an approximate continuity point of $\nabla_{\mathrm{H}} u$ concludes the proof.

The next theorem has been proved by Calderón and Zygmund for functions of bounded variation in Euclidean spaces [6] (see also [16]).

THEOREM 2.3 (Calderón-Zygmund). Let $u \in \operatorname{BV}_{\mathrm{H}, \mathrm{loc}}(\Omega)$ and let $\beta=$ $Q /(Q-1)$. Then $u$ is $L^{\beta}$ differentiable of order 1 at a.e. $x \in \Omega$ and the differential is representable as $\nabla_{\mathrm{H}} u(x)^{*}$, where $\nabla_{\mathrm{H}} u$ is the density of $D_{\mathrm{H}}^{\mathrm{a}} u$.

Proof. Due to Lemma 2.2, for a.e. $x \in A_{u} \cap A_{\nabla_{\mathrm{H}} u}$ we have

$$
\lim _{r \rightarrow 0^{+}} \frac{\left|D_{\mathrm{H}} v\right|\left(B_{x, r}\right)}{r^{Q}}=0,
$$

where $v(y)=u(y)-\nabla_{\mathrm{H}} u(x)^{*}\left(x^{-1} y\right)-u(x)$ and $x \in A_{v}$ with $v(x)=0$. By the triangle inequality we have

$$
\left(f_{B_{x, r}}|v(y)|^{\beta} d y\right)^{1 / \beta} \leq\left(f_{B_{x, r}}\left|v(y)-v_{B_{x, r}}\right|^{\beta} d y\right)^{1 / \beta}+\left|v_{B_{x, r}}\right| .
$$

The Sobolev-Poincaré inequality (9) and the estimate (20) give a constant $C_{1}$ such that

$$
\begin{aligned}
& \left(\underset{B_{x, r}}{f}|v(y)|^{\beta} d y\right)^{1 / \beta} \\
& \quad \leq C_{1} r\left\{\int_{0}^{1} \frac{\left|D_{\mathrm{H}} v\right|\left(B_{x, t r}\right)}{(t r)^{Q}} d t+\frac{\left|D_{\mathrm{H}} v\right|\left(B_{x, r}\right)}{r^{Q}}\right\}=o(r),
\end{aligned}
$$

where the last equality follows from condition (24).

The approach used in the previous theorem can be applied to $L^{p}$ differentiability of Sobolev functions, obtaining the following known results (see $[3])$. 
THEOREM 2.4. Let $u \in W_{\mathrm{H}, \mathrm{loc}}^{1, p}(\Omega)$ with $1 \leq p<Q$. Then $u$ has an $L^{\gamma}$ differential of order 1 at a.e. $x \in \Omega$ and the differential is representable as $\nabla_{\mathrm{H}} u(x)^{*}$, where $\nabla_{\mathrm{H}} u$ is the density of $D_{\mathrm{H}}^{\mathrm{a}} u$ and $\gamma=Q p /(Q-p)$.

THEOREM 2.5. Let $u \in W_{\mathrm{H}, \mathrm{loc}}^{1, p}(\Omega)$ with $1 \leq p<\infty$. Then $u$ has an $L^{p}$ differential of order 1 at a.e. $x \in \Omega$ and the differential is representable as $\nabla_{\mathrm{H}} u(x)^{*}$, where $\nabla_{\mathrm{H}} u$ is the density of $D_{\mathrm{H}}^{\mathrm{a}} u$.

2.1. Another proof of Rademacher's theorem. As a consequence of a.e. $L^{1}$ differentiability of H-BV functions we obtain another proof of the Rademacher theorem for real-valued Lipschitz maps on stratified groups. This result for group-valued maps is much more demanding and it is due to Pansu [39]. In the case of real-valued Lipschitz maps on Carnot-Carathéodory spaces, this theorem has been proved by Monti and Serra Cassano [38], using a Morreytype estimate in Carnot-Carathéodory spaces due to Lu [33]. In the general case of doubling metric spaces admitting the Poincare inequality, another proof of Rademacher's theorem is given in [3] for functions belonging to $W_{\mathrm{H}}^{1, p}(\Omega)$, where $p$ is greater than the homogeneous dimension of the metric space. In stratified groups, a different proof of this theorem is given in [14].

Here we present a different approach which applies Lemma 3.1.5 of [16]. By this lemma, the Rademacher theorem follows by a.e. approximate differentiability. Our proof extends this lemma to stratified groups and uses the a.e. $L^{1}$ differentiability of functions of $\mathrm{H}$-bounded variation, proved in Theorem 2.3.

ThEOREM 2.6 (Rademacher). Let $u: \Omega \rightarrow \mathbb{R}$ be a Lipschitz map. Then for a.e. $x \in \Omega$ there exists a unique vector $\nabla_{\mathrm{H}} u(x) \in V_{1}$ such that

$$
\lim _{y \rightarrow x} \frac{\left|u(y)-u(x)-\nabla_{\mathrm{H}} u(x)^{*}\left(x^{-1} y\right)\right|}{\varrho(x, y)}=0 .
$$

Proof. It is not difficult to check that $u$ has distributional derivative $X_{j} u$ in $L^{\infty}(\Omega)$ for every $j=1, \ldots, m$ (see [21] and [23] for more information). In particular $u \in \operatorname{BV}_{\mathrm{H}}(\Omega)$ and Theorem 2.3 largely ensures that $u$ is $L^{1}$ differentiable at a.e. $x \in \Omega$. Let us pick $x \in \Omega$ at which $u$ is $L^{1}$ differentiable with differential $\nabla_{\mathrm{H}} u(x)^{*}$. By Lemma 1.16 with $\alpha=1$ we obtain the limit

$$
\lim _{r \rightarrow 0^{+}} \underset{B_{x, r}}{f} \frac{\left|u(y)-u(x)-\nabla_{\mathrm{H}} u(x)^{*}\left(x^{-1} y\right)\right|}{\varrho(x, y)} d y=0 .
$$

In particular $u$ is approximately differentiable at $x$ in the Federer sense [16], that is, for every $\varepsilon>0$ the set

$$
E_{\varepsilon}=\left\{y \in \Omega|| u(y)-u(x)-\nabla_{\mathrm{H}} u(x)^{*}\left(x^{-1} y\right) \mid \leq \varepsilon \varrho(x, y)\right\}
$$

has the property $\left|E_{\varepsilon} \cap B_{x, r}\right|\left|B_{x, r}\right|^{-1} \rightarrow 1$ as $r \rightarrow 0^{+}$. Then $x$ is a density point of $E_{\varepsilon}$, hence the doubling property gives that $\operatorname{dist}\left(y, E_{\varepsilon}\right)=o(\varrho(y, x))$ as 
$\varrho(y, x) \rightarrow 0^{+}$. Fix $r_{0}>0$ such that $\operatorname{dist}\left(y, E_{\varepsilon}\right) \leq \varepsilon \varrho(y, x)$ for every $\varrho(y, x) \leq$ $r_{0}$. We choose $y \in \bar{B}_{x, r_{0}}$, assuming that $\bar{B}_{x, r_{0}} \subset \Omega$. If $y \notin E_{\varepsilon} \cap \bar{B}_{x, r_{0}} \subset \Omega$, then $\operatorname{dist}\left(y, E_{\varepsilon}\right)>0$ and there exists $a \in E_{\varepsilon}$ such that $\varrho(y, a)<2 \operatorname{dist}\left(y, E_{\varepsilon}\right)$. We denote by $L$ the Lipschitz constant of $u$. We have

$$
\begin{aligned}
\mid u(y) & -u(x)-\nabla_{\mathrm{H}} u(x)^{*}\left(x^{-1} y\right) \mid \\
& \leq\left|\nabla_{\mathrm{H}} u(x)^{*}\left(a^{-1} y\right)\right|+\left|u(a)-u(x)-\nabla_{\mathrm{H}} u(x)^{*}\left(x^{-1} a\right)\right|+|u(y)-u(a)| \\
& \leq\left\|\nabla_{\mathrm{H}} u(x)^{*}\right\| 2 \varepsilon \varrho(y, x)+\varepsilon \varrho(y, x)+2 L \varepsilon \varrho(y, x) .
\end{aligned}
$$

In the case $y \in E_{\varepsilon} \cap \bar{B}_{x, r_{0}}$, by definition of $E_{\varepsilon}$, we have $\mid u(y)-u(x)-$ $\nabla_{\mathrm{H}} u(x)^{*}\left(x^{-1} y\right) \mid \leq \varepsilon \varrho(y, x)$. The arbitrary choice of $\varepsilon$ concludes the proof.

3. Higher order differentiability. In the first part of this section we review some basic facts about left invariant differential operators on groups. In particular we recall the important Poincaré-Birkhoff-Witt theorem, which will be of importance to our differentiability results of higher order.

In $\mathbb{R}^{n}$ for every $k \in \mathbb{N} \backslash\{0\}$ there is a natural correspondence between differential operators $\partial_{x}^{\alpha}$ with $|\alpha|=\sum_{j=1}^{n} \alpha_{j} \leq k$ and polynomials of degree less than or equal to $k$. The same analogy holds for stratified groups. For convenience of notation, we will identify the Lie algebra $\mathcal{G}$ of left invariant vector fields with the isomorphic vector space of first order left invariant differential operators. Let $\left(W_{1}, \ldots, W_{q}\right)$ be a graded basis of $\mathcal{G}$, regarded as a basis of first order left invariant differential operators. Recall that a homogeneous left invariant differential operator $Z$ has degree $d$ if $\delta_{r}^{*} Z=r^{d} Z$, that is,

$$
\delta_{r}^{*} Z(\varphi)=Z\left(\varphi \circ \delta_{r}\right)=d \varphi\left(\delta_{r} Z\right)=r^{d} d \varphi(Z)=r^{d} Z \varphi
$$

for every $r>0$ and $\varphi \in C^{\infty}(\mathbb{G})$. By this definition one easily checks that the left invariant vector field $W_{i}$ of degree $d_{i}$ also has homogeneous degree $d_{i}$ as left invariant differential operator. With this notion we can define the homogeneous degree of a left invariant differential operator. We consider a left invariant differential operator

$$
Z=\sum_{\alpha} c_{\alpha} W^{\alpha},
$$

where only a finite number of coefficients $c_{\alpha} \in \mathbb{R}$ do not vanish and $W^{\alpha}$ is defined by the following ordered compositions:

$$
W^{\alpha}=W_{1}^{\alpha_{1}} \cdots W_{q}^{\alpha_{q}}
$$

for every $\alpha=\left(\alpha_{1}, \ldots, \alpha_{q}\right) \in \mathbb{N}^{q}$. We define the homogeneous degree of $Z$ as

$$
\mathrm{h}-\operatorname{deg}(Z)=\max \left\{d(\alpha) \mid c_{\alpha} \neq 0\right\},
$$

where $d(\alpha)=\sum_{k=1}^{q} d_{k} \alpha_{k}$. The finite-dimensional space of left invariant differential operators of homogeneous degree less than or equal to $k$ will be 
denoted by $\mathcal{A}_{k}(\mathbb{G})$. The Poincaré-Birkhoff-Witt theorem states that the set of differential operators

$$
\left\{W_{1}^{\alpha_{1}} \cdots W_{q}^{\alpha_{q}} \mid\left(\alpha_{1}, \ldots, \alpha_{q}\right) \in \mathbb{N}^{q}\right\}
$$

is a basis of the algebra of left invariant differential operators on $\mathbb{G}$ (see p. 21 of [17]). The next proposition states a precise relation between polynomials and left invariant differential operators on groups (see Proposition 1.30 of [17]).

Proposition 3.1. There exists an isomorphism $L: \mathcal{P}_{\mathrm{H}, k}(\mathbb{G}) \rightarrow \mathcal{A}_{k}(\mathbb{G})$, given by

$$
L(P)=\sum_{d(\alpha) \leq k}\left(W^{\alpha} P\right)(0) W^{\alpha} .
$$

Definition 3.2 (Mixed derivatives). Let $u \in \mathrm{BV}_{\mathrm{H}}^{k}(\Omega)$ and let $Y_{1}, \ldots, Y_{p}$ $\in \mathcal{G}$ be first order left invariant differential operators with $\sum_{j=1}^{p} \mathrm{~h}-\operatorname{deg}\left(Y_{j}\right)$ $\leq k$. We define the Radon measure $D_{Y_{1} \cdots Y_{p}} u$ as follows:

$$
\int_{\Omega} \phi d D_{Y_{1} \cdots Y_{p}} u=(-1)^{p} \int_{\Omega} u(x)\left(Y_{p} \cdots Y_{1} \phi\right)(x) d x \quad \forall \phi \in C_{\mathrm{c}}^{\infty}(\Omega) .
$$

Some remarks here are in order. Since the flow associated to $Y \in \mathcal{G}$ preserves the measure, we infer that $\operatorname{div}(Y)=0$, hence by the classical divergence theorem relation (26) holds for smooth functions $u$ with $D_{Y_{1} \cdots Y_{p}} u=$ $\left(Y_{1} \cdots Y_{p} u\right) v_{g}$.

Note also that the existence of the distributional derivative $D_{Y_{1} \cdots Y_{p}} u$ as a measure follows by the Lie bracket generating condition on horizontal vector fields $\left(X_{1}, \ldots, X_{m}\right)$ that are now regarded as differential operators. It suffices to observe that every left invariant differential operator $Z$ with h-deg $(Z)=k$ can be written as a linear combination of up to $k$-fold iterated commutators of $\left(X_{1}, \ldots, X_{m}\right)$. Then one uses the definition of $\mathrm{H}-\mathrm{BV}^{k}$ functions, which exactly requires that every iterated distributional derivative $X_{j_{1}} \cdots X_{j_{k}} u$ is a measure, where $1 \leq j_{1}, \ldots, j_{k} \leq m$.

By the Radon-Nikodým theorem we have $D_{Y_{1}, \ldots, Y_{p}} u=\left(D_{Y_{1}, \ldots, Y_{p}} u\right)^{\mathrm{a}}+$ $\left(D_{Y_{1}, \ldots, Y_{p}} u\right)^{\mathrm{s}}$, where the terms of the sum are the absolutely continuous part and the singular part of $D_{Y_{1} \cdots Y_{p}} u$, respectively. The density of $\left(D_{Y_{1} \cdots Y_{p}} u\right)^{\mathrm{a}}$ will be denoted simply by $Y_{1} \cdots Y_{p} u$. In the case the measure $\left(D_{Y_{1} \cdots Y_{p}} u\right)^{\mathrm{a}}$ is replaced by $\left(D_{W^{\alpha}} u\right)^{\mathrm{a}}$ its density will be denoted by $W^{\alpha} u$. Recall that for every $i=1, \ldots, m$ the differential operators $W_{i} \in \mathcal{G}$ have homogeneous degree equal to one. We will denote these operators by $X_{i}$, where $i=1, \ldots, m$.

Due to the Poincaré-Birkhoff-Witt theorem, for every $j_{1}, \ldots, j_{p} \in$ $\{1, \ldots, m\}$ there exist coefficients $\left\{c_{j_{1}, \ldots, j_{p}}^{\alpha_{1}, \ldots, \alpha_{q}}\right\}$ such that

$$
X_{j_{1}} \cdots X_{j_{p}}=\sum_{d(\alpha) \leq p} c_{j_{1}, \ldots, j_{p}}^{\alpha_{1}, \ldots, \alpha_{q}} W_{1}^{\alpha_{1}} \cdots W_{q}^{\alpha_{q}}
$$


where $\alpha=\left(\alpha_{1}, \ldots, \alpha_{q}\right)$ and $X_{j_{s}}$ is a horizontal differential operator for every $s=1, \ldots, p$, that is, it has homogeneous degree equal to one. By a direct computation, it is not difficult to see that Definition 3.2 and the linearity of (27) give the relation

$$
D_{X_{j_{1}} \ldots X_{j_{p}}} u=(-1)^{p} \sum_{d(\alpha) \leq p}(-1)^{|\alpha|} c_{j_{p}, \ldots, j_{1}}^{\alpha_{1}, \ldots, \alpha_{q}} D_{W_{1}^{\alpha_{1} \ldots W_{q}}} u
$$

for every $u \in \operatorname{BV}_{\mathrm{H}}^{k}(\Omega)$ and $p \leq k$.

3.1. Differentiability of $\mathrm{H}-\mathrm{BV}^{k}$ functions. Here we prove the higher order approximate differentiability of $\mathrm{H}_{-} \mathrm{BV}^{k}$ functions. We start with a preliminary lemma.

Lemma 3.3. Let $u \in \mathrm{BV}_{\mathrm{H}, \mathrm{loc}}^{k}(\Omega)$. Then for a.e. $x \in \bigcap_{d(\alpha) \leq k} A_{W^{\alpha} u}$ there exists a unique polynomial $P_{[x]} \in \mathcal{P}_{\mathrm{H}, k}(\mathbb{G})$ such that $W^{\alpha} P_{[x]}(x)=W^{\alpha} u(x)$ and

$$
\lim _{r \rightarrow 0^{+}}\left|D_{W^{\alpha}}\left(u-P_{[x]}\right)\right|\left(B_{x, r}\right) r^{-Q}=0 \quad \text { for every } d(\alpha) \leq k .
$$

Proof. By Proposition 3.1 for every $x \in \bigcap_{d(\alpha) \leq k} A_{W^{\alpha} u}$ we have a unique polynomial $P_{[x]} \in \mathcal{P}_{\mathrm{H}, k}(\mathbb{G})$ such that $W^{\alpha} P_{[x]}(x)=W^{\alpha} u(x)$ for all $d(\alpha) \leq k$. By the regularity of $P_{[x]}$ we have the decomposition

$$
\left|D_{W^{\alpha}}\left(u-P_{[x]}\right)\right|=\left|W^{\alpha} u-W^{\alpha} P_{[x]}\right| v_{g}+\left|D_{W^{\alpha}} u\right|^{\mathrm{s}} .
$$

Then Lemma 2.1 concludes the proof.

TheOREM 3.4. Let $u \in \mathrm{BV}_{\mathrm{H}, \mathrm{loc}}^{k}(\Omega)$ with $1 \leq k<Q$ and define $\gamma=$ $Q /(Q-k)$. Then $u$ is a.e. $L^{\gamma}$ differentiable of order $k$.

Proof. By Lemma 3.3 for a.e. $x \in \bigcap_{d(\alpha) \leq k} A_{W^{\alpha} u}$ we have a unique polynomial $P_{[x]} \in \mathcal{P}_{\mathrm{H}, k}(\mathbb{G})$ such that $W^{\alpha} P_{[x]}(x)=W^{\alpha} u(x)$ and

$$
\lim _{r \rightarrow 0^{+}}\left|D_{W^{\alpha}}\left(u-P_{[x]}\right)\right|\left(B_{x, r}\right) r^{-Q}=0
$$

for every $d(\alpha) \leq k$. By induction on $k$, suppose that our claim is satisfied for $k-1 \geq 1$ and that $u \in L_{\mathrm{loc}}^{\eta}(\Omega)$, where $\eta=Q /(Q-k+1)$. Theorem 2.3 shows that our claim holds for $k-1=1$. For every $j=1, \ldots, m$ the functions $X_{j} u$ and $u$ are $\mathrm{H}-\mathrm{BV}^{k-1}$, so the induction hypothesis implies that $u, X_{j} u \in$ $L_{\text {loc }}^{\eta}(\Omega)$ and there exists a unique polynomial $R_{j} \in \mathcal{P}_{\mathrm{H}, k-1}(\mathbb{G})$ such that

$$
\left(f_{B_{x, r}}\left|X_{j} u(y)-R_{j}(y)\right|^{\eta} d y\right)^{1 / \eta}=o\left(r^{k-1}\right) \quad \text { as } r \rightarrow 0^{+},
$$

and $W^{\beta} X_{j} u(x)=W^{\beta} R_{j}(x)$ for every $d(\beta) \leq k-1$. By the PoincaréBirkhoff-Witt theorem we have a finite number of coefficients $\left\{c_{\beta, j}^{\alpha}\right\}$ such 
that

$$
\begin{aligned}
W^{\beta} X_{j} u(x) & =\sum_{d(\alpha) \leq k} c_{\beta, j}^{\alpha} W^{\alpha} u(x)=\sum_{d(\alpha) \leq k} c_{\beta, j}^{\alpha} W^{\alpha} P_{[x]}(x) \\
& =W^{\beta} X_{j} P_{[x]}(x),
\end{aligned}
$$

so $W^{\beta} R_{j}(x)=W^{\beta} X_{j} P_{[x]}(x)$ for every $d(\beta) \leq k-1$. By Proposition 3.1, observing that $X_{j} P_{[x]} \in \mathcal{P}_{\mathrm{H}, k-1}(\mathbb{G})$ we have $R_{j}=X_{j} P_{[x]}$ for every $j=$ $1, \ldots, m$. Thus for every $j=1, \ldots, m$ we have $X_{j}\left(u-P_{[x]}\right)=X_{j} u-R_{j}$. As a result, defining $v=u-P_{[x]}$ and using condition (29) for every $j=1, \ldots, m$ we obtain

$$
\left(f_{B_{x, r}}\left|X_{j} v(y)\right|^{\eta} d y\right)^{1 / \eta}=o\left(r^{k-1}\right) .
$$

The condition $k<Q$ implies the inequality $\eta=Q(Q-k+1)^{-1}<Q$, hence the Sobolev-Poincaré inequality (8) yields

$$
\left(f_{B_{x, r}}\left|v(y)-v_{B_{x, r}}\right|^{\gamma} d y\right)^{1 / \gamma} \leq C r\left(f_{B_{x, r}}\left|\nabla_{\mathrm{H}} v(y)\right|^{\eta} d y\right)^{1 / \eta}
$$

where $\gamma=Q \eta /(Q-\eta)=Q /(Q-k)$. From condition (31) for every $j=$ $1, \ldots, m$ we obtain

$$
\left(f_{B_{x, r}}\left|v(y)-v_{B_{x, r}}\right|^{\gamma} d y\right)^{1 / \gamma}=o\left(r^{k}\right)
$$

Notice that $v \in W_{\mathrm{H}, \mathrm{loc}}^{1, \eta}(\Omega)$, so we can apply $(21)$ to get

$$
\left|v_{B_{x, r}}\right| \leq C r\left\{\int_{0}^{1}\left(f_{B_{x, t r}}\left|\nabla_{\mathrm{H}} v(y)\right|^{\eta}\right)^{1 / \eta} d t+\left(f_{B_{x, r}}\left|\nabla_{\mathrm{H}} u(y)\right|^{\eta}\right)^{1 / \eta}\right\}
$$

hence condition (31) gives $\left|v_{B_{x, r}}\right|=o\left(r^{k}\right)$ as $r \rightarrow 0^{+}$. Finally, by the triangle inequality we have

$$
\left(f_{B_{x, r}}|v(y)|^{\gamma} d y\right)^{1 / \gamma} \leq\left(f_{B_{x, r}}\left|v(y)-v_{B_{x, r}}\right|^{\gamma} d y\right)^{1 / \gamma}+\left|v_{B_{x, r}}\right|=o\left(r^{k}\right)
$$

thereby finishing the proof.

The previous proof by induction works for $\mathrm{H}-\mathrm{BV}^{k}$ with arbitrary $k$, provided that the use of (8) and (21) is replaced by that of (10) and (20), respectively. As a result, we obtain the following theorem.

THEOREM 3.5. Every $u$ belonging to $\mathrm{BV}_{\mathrm{H}, \mathrm{loc}}^{k}(\Omega)$ is a.e. $L^{1}$ differentiable of order $k$. 
3.2. Differentiability of $H$-Sobolev functions. We first observe that $W_{\mathrm{H}, \text { loc }}^{k, p}(\Omega) \subset \mathrm{BV}_{\mathrm{H} \text {,loc }}^{k}(\Omega)$, thus all previous considerations for $\mathrm{H}-\mathrm{BV}^{k}$ functions hold for H-Sobolev functions of the same order. We have in addition $D_{W^{\alpha}} u=\left(W^{\alpha} u\right) v_{g}$, where $W^{\alpha} u \in L^{p}(\Omega)$ for every $d(\alpha) \leq k$.

Lemma 3.6. Let $u \in W_{\mathrm{H}, \mathrm{loc}}^{k, p}(\Omega)$. Then for a.e. $x \in \bigcap_{d(\alpha) \leq k} A_{W^{\alpha} u}$ there exists a unique polynomial $P_{[x]} \in \mathcal{P}_{\mathrm{H}, k}(\mathbb{G})$ such that $W^{\alpha} P_{[x]}(x)=W^{\alpha} u(x)$ and

$$
\lim _{r \rightarrow 0^{+}} f_{B_{x, r}}\left|W^{\alpha} u(y)-W^{\alpha} P_{[x]}(y)\right|^{p} d y=0
$$

for every $d(\alpha) \leq k$.

Proof. By Propositions 1.10 and 3.1 for a.e. $x \in \Omega$ we have a unique polynomial $P_{[x]} \in \mathcal{P}_{\mathrm{H}, k}(\mathbb{G})$ such that $W^{\alpha} P_{[x]}(x)=W^{\alpha} u(x)$ for every $d(\alpha) \leq k$, where the number $W^{\alpha} u(x)$ has the property

$$
\lim _{r \rightarrow 0^{+}} \underset{B_{x, r}}{f}\left|W^{\alpha} u(y)-W^{\alpha} P_{[x]}(x)\right|^{p} d y=0
$$

for every $d(\alpha) \leq k$. Then the continuity of $W^{\alpha} P_{[x]}$ leads us to the conclusion.

THEOREM 3.7. Let $u \in W_{\mathrm{H}, \mathrm{loc}}^{k, p}(\Omega)$ with $1 \leq k p<Q$ and define $\gamma=$ $Q p /(Q-k p)$. Then $u$ is a.e. $L^{\gamma}$ differentiable of order $k$.

Proof. By Lemma 3.6 for a.e. $x \in \Omega$ we have a unique polynomial $P_{[x]} \in$ $\mathcal{P}_{\mathrm{H}, k}(\mathbb{G})$ such that $W^{\alpha} P_{[x]}(x)=W^{\alpha} u(x)$ and

$$
\lim _{r \rightarrow 0^{+}} \underset{B_{x, r}}{f}\left|W^{\alpha} u(y)-W^{\alpha} P_{[x]}(y)\right|^{p} d y=0
$$

for every $d(\alpha) \leq k$. By induction on $k$, suppose that our claim is satisfied for $k-1 \geq 1$ and that $u \in L_{\text {loc }}^{\eta}(\Omega)$, where $\eta=Q p(Q-(k-1) p)^{-1}$. Theorem 2.4 and the Sobolev-Poincaré inequality (8) ensure that our claim holds for $k-1=1$. We have $u, X_{j} u \in W_{\mathrm{H}}^{k-1, p}(\Omega)$ for every $j=1, \ldots, m$. By the induction hypothesis, $u, X_{j} u \in L_{\text {loc }}^{\eta}(\Omega)$ and for every $j=1, \ldots, m$ there exists a unique polynomial $R_{j} \in \mathcal{P}_{\mathrm{H}, k-1}(\mathbb{G})$ such that

$$
\left(f_{B_{x, r}}\left|X_{j} u(y)-R_{j}(y)\right|^{\eta} d y\right)^{1 / \eta}=o\left(r^{k-1}\right),
$$

which satisfies the condition $W^{\beta} X_{j} u(x)=W^{\beta} R_{j}(x)$ for every $d(\beta) \leq k-1$. By the Poincaré-Birkhoff-Witt theorem we have a finite number of coefficients $\left\{c_{\beta, j}^{\alpha}\right\}$ such that

$$
W^{\beta} X_{j} u(x)=\sum_{d(\alpha) \leq k} c_{\beta, j}^{\alpha} W^{\alpha} u(x)=\sum_{d(\alpha) \leq k} c_{\beta, j}^{\alpha} W^{\alpha} P_{[x]}=W^{\beta} X_{j} P_{[x]}(x),
$$


so $W^{\beta} R_{j}(x)=W^{\beta} X_{j} P_{[x]}(x)$ for every $d(\beta) \leq k-1$. In view of Proposition 3.1 and observing that $X_{j} P_{[x]} \in \mathcal{P}_{\mathrm{H}, k-1}(\mathbb{G})$ we have $R_{j}=X_{j} P_{[x]}$ for every $j=1, \ldots, m$. Then, defining $v=u-P_{[x]}$ we obtain $X_{j} v=X_{j} u-R_{j}$ for every $j=1, \ldots, m$. Thus, relation (34) yields

$$
\left(f_{B_{x, r}}\left|X_{j} v(y)\right|^{\eta} d y\right)^{1 / \eta}=o\left(r^{k-1}\right) .
$$

Then we arrive at the condition

$$
\left(\underset{B_{x, r}}{f}\left|\nabla_{\mathrm{H}} v(y)\right|^{\eta} d y\right)^{1 / \eta}=o\left(r^{k-1}\right) .
$$

Recall that by induction hypothesis $v \in W_{\mathrm{H}}^{1, \eta}(\Omega)$, furthermore the condition $k p<Q$ gives $\eta<Q$; therefore we can apply the Sobolev-Poincaré inequality (8) to get

$$
\left(f_{B_{x, r}}|v(y)|^{\gamma} d y\right)^{1 / \gamma} \leq C r\left(f_{B_{x, r}}\left|\nabla_{\mathrm{H}} v(y)\right|^{\eta} d y\right)^{1 / \eta}+\left|v_{B_{x, r}}\right|,
$$

where $\gamma=Q \eta /(Q-\eta)=Q p /(Q-k p)$. Moreover, by (21) we have the estimate

$$
\left|v_{B_{x, r}}\right| \leq C r\left\{\int_{0}^{1}\left(f_{B_{x, t r}}\left|\nabla_{\mathrm{H}} u(y)\right|^{\eta} d y\right)^{1 / \eta} d t+\left(\underset{B_{x, r}}{f_{\mathrm{H}}}\left|\nabla_{\mathrm{H}} u(y)\right|^{\eta} d y\right)^{1 / \eta}\right\} .
$$

Then there exists a constant $C_{1}$ such that

$$
\begin{aligned}
& \left(\underset{B_{x, r}}{f}|v(y)|^{\gamma} d y\right)^{1 / \gamma} \\
& \quad \leq C_{1} r\left\{\int_{0}^{1}\left(f_{B_{x, t r}}\left|\nabla_{\mathrm{H}} u(y)\right|^{\eta} d y\right)^{1 / \eta} d t+\left({\underset{B}{B_{x, r}}}\left|\nabla_{\mathrm{H}} u(y)\right|^{\eta} d y\right)^{1 / \eta}\right\} .
\end{aligned}
$$

Finally, relation (35) and the previous inequality conclude the proof.

Replacing in the previous proof the Sobolev-Poincaré inequality (8) with the Poincaré inequality (7) and Theorem 2.4 with Theorem 2.5, we obtain the following theorem.

THEOREM 3.8. Every function u belonging to $W_{\mathrm{H}, \mathrm{loc}}^{k, p}(\Omega)$ is a.e. $L^{p}$ differentiable of order $k$.

\section{References}

[1] L. Ambrosio and V. Magnani, Weak differentiability of $B V$ functions on stratified groups, Math. Z. 245 (2003), 123-153. 
[2] T. Bagby and W. P. Ziemer, Pointwise differentiability and absolute continuity, Trans. Amer. Math. Soc. 19 (1974), 129-148.

[3] J. Björn, $L^{q}$-differentials for weighted Sobolev spaces, Michigan Math. J. 47 (2000), 151-161.

[4] B. Bojarski and P. Hajłasz, Pointwise inequalities for Sobolev functions and some applications, Studia Math. 106 (1993), 77-92.

[5] B. Bojarski, P. Hajłasz and P. Strzelecki, Improved $C^{k, \lambda}$ approximation of higher order Sobolev functions in norm and capacity, Indiana Univ. Math. J. 51 (2002), 507-540.

[6] A. P. Calderón and A. Zygmund, On the differentiability of functions which are of bounded variation in Tonelli's sense, Rev. Unión Mat. Argentina 20 (1960), 103-121.

[7] - - - Local properties of solutions of elliptic partial differential equations, Studia Math. 20 (1961), 179-255.

[8] C. P. Calderón, E. B. Fabes and N. M. Rivière, Maximal smoothing operators, Indiana Univ. Math. J. 23 (1974), 889-897.

[9] L. Capogna, D. Danielli and N. Garofalo, The geometric Sobolev embedding for vector fields and the isoperimetric inequality, Comm. Anal. Geom. 2 (1994), 203215.

[10] - , - - Subelliptic mollifiers and a basic pointwise estimate of Poincaré type, Math. Z. 226 (1997), 147-154.

[11] A. Córdoba, Bounded variation and differentiability of functions, Collect. Math. 26 (1975), 227-238.

[12] L. Corwin and F. P. Greenleaf, Representations of Nilpotent Lie Groups and Their Applications. Part 1: Basic Theory and Examples, Cambridge Univ. Press, 1990.

[13] D. Danielli, N. Garofalo and D. M. Nhieu, Notions of convexity in Carnot groups, Comm. Anal. Geom. 11 (2003), 263-341.

[14] D. Danielli, N. Garofalo and S. Salsa, Variational inequalities with lack of ellipticity. I. Optimal interior regularity and non-degeneracy of the free boundary, Indiana Univ. Math. J. 52 (2003), 361-398.

[15] J. R. Dorronsoro, Differentiability properties of functions with bounded variation, ibid. 38 (1989), 1027-1045.

[16] H. Federer, Geometric Measure Theory, Springer, 1969.

[17] G. B. Folland and E. M. Stein, Hardy Spaces on Homogeneous Groups, Princeton Univ. Press, 1982.

[18] B. Franchi, G. Lu and R. L. Wheeden, Representation formulas and weighted Poincaré inequalities for Hörmander vector fields, Ann. Inst. Fourier (Grenoble) 45 (1995), 577-604.

[19] - , - - , A relationship between Poincaré inequalities and representation formulas in spaces of homogeneous type, Int. Math. Res. Not. 1996, no. 1, 1-14.

[20] B. Franchi, R. Serapioni and F. Serra Cassano, Meyers-Serrin type theorems and relaxation of variational integrals depending on vector fields, Houston J. Math. 22 (1996), 859-889.

[21] - , 一, - Approximation and imbedding theorems for weighted Sobolev spaces associated with Lipschitz continuous vector fields, Boll. Un. Mat. Ital. B (7) 11 (1997), 83-117.

[22] N. Garofalo and D. M. Nhieu, Isoperimetric and Sobolev inequalities for CarnotCarathéodory spaces and the existence of minimal surfaces, Comm. Pure Appl. Math. 49 (1996), 1081-1144.

[23] - - - Lipschitz continuity, global smooth approximation and extension theorems for Sobolev functions in Carnot-Carathéodory spaces, J. Anal. Math. 74 (1998), 67-97. 
[24] N. Garofalo and F. Tournier, The theorem of Busemann-Feller-Alexandrov in Carnot groups, Comm. Anal. Geom. 12 (2004), 853-886.

[25] M. Gromov, Carnot-Carathéodory spaces seen from within, in: Sub-Riemannian Geometry, A. Bellaiche and J. Risler (eds.), Progr. Math. 144, Birkhäuser, Basel, 1996, 79-323.

[26] C. E. Gutiérrez and A. Montanari, Maximum and comparison principles for convex functions on the Heisenberg group, Comm. Partial Differential Equations 29 (2004), 1305-1334.

[27] P. Hajłasz and P. Koskela, Sobolev met Poincaré, Mem. Amer. Math. Soc. 145 (2000), no. 688.

[28] D. Jerison, The Poincaré inequality for vector fields satisfying Hörmander's condition, Duke Math. 53 (1986), 503-523.

[29] E. Lanconelli and D. Morbidelli, On the Poincaré inequality for vector fields, Ark. Mat. 38 (2000), 327-342.

[30] F. C. Liu, A Luzin type property of Sobolev functions, Indiana Univ. Math. J. 26 (1977), 645-651.

[31] G. Lu, Weighted Poincaré and Sobolev inequalities for vector fields satisfying Hörmander's condition and applications, Rev. Mat. Iberoamericana 8 (1992), 367-439.

[32] - , The sharp Poincaré inequality for free vector fields: an endpoint result, ibid. 10 (1994), 453-466.

[33] - Embedding theorems into Lipschitz and BMO spaces and applications to quasilinear subelliptic differential equations, Publ. Mat. 40 (1996), 301-329.

[34] G. Lu, J. Manfredi and B. Stroffolini, Convex functions on the Heisenberg group, Calc. Var. Partial Differential Equations 19 (2004), 1-22.

[35] G. Lu and R. L. Wheeden, An optimal representation formula for Carnot-Carathéodory vector fields, Bull. London Math. Soc. 30 (1998), 578-584.

[36] V. Magnani, Lipschitz continuity, Aleksandrov theorem and characterizations for H-convex functions, preprint, 2003.

[37] P. Maheux et L. Saloff-Coste, Analyse sur les boules d'un opérateur sous-elliptique, Math. Ann. 303 (1995), 713-740.

[38] R. Monti and F. Serra Cassano, Surface measures in Carnot-Carathéodory spaces Calc. Var. Partial Differential Equations 13 (2001), 339-376.

[39] P. Pansu, Métriques de Carnot-Carathéodory et quasiisométries des espaces symétriques de rang un, Ann. of Math. 129 (1989), 1-60.

[40] L. Saloff-Coste, Aspects of Sobolev-Type Inequalities, Cambridge Univ. Press, Cambridge, 2002.

[41] E. M. Stein, Singular Integrals and Differentiability Properties of Functions, Princeton Univ. Press, Princeton, NJ, 1970.

[42] -, Harmonic Analysis, Princeton Univ. Press, 1993.

[43] N. Th. Varopoulos, Fonctions harmoniques sur les groupes de Lie, C. R. Acad. Sci. Paris Sér. I Math. 304 (1987), 519-521.

[44] N. Th. Varopoulos, L. Saloff-Coste and T. Coulhon, Analysis and Geometry on Groups, Cambridge Univ. Press, Cambridge, 1992.

[45] S. K. Vodop'yanov, $\mathcal{P}$-Differentiability on Carnot groups in different topologies and related topics, in: Proc. Anal. Geom., Sobolev Institute Press, Novosibirsk, 2000, 603-670. 
[46] W. P. Ziemer, Weakly Differentiable Functions, Springer, 1989.

Dipartimento di Matematica

Università di Pisa

via Buonarroti n. 2, 56127 Pisa, Italy

E-mail: magnani@dm.unipi.it

Received January 27, 2004

Revised version January 17, 2005 